\title{
The EH Interpolation Spline and Its Approximation
}

\author{
Jin Xie ${ }^{1}$ and Xiaoyan Liu ${ }^{2}$ \\ ${ }^{1}$ Department of Mathematics and Physics, Hefei University, Hefei 230601, China \\ ${ }^{2}$ Department of Mathematics, University of La Verne, La Verne, CA 91750, USA
}

Correspondence should be addressed to Jin Xie; hfuuxiejin@126.com

Received 28 February 2014; Accepted 15 June 2014; Published 30 June 2014

Academic Editor: Grzegorz Nowak

Copyright (C) 2014 J. Xie and X. Liu. This is an open access article distributed under the Creative Commons Attribution License, which permits unrestricted use, distribution, and reproduction in any medium, provided the original work is properly cited.

\begin{abstract}
A new interpolation spline with two parameters, called EH interpolation spline, is presented in this paper, which is the extension of the standard cubic Hermite interpolation spline, and inherits the same properties of the standard cubic Hermite interpolation spline. Given the fixed interpolation conditions, the shape of the proposed splines can be adjusted by changing the values of the parameters. Also, the introduced spline could approximate to the interpolated function better than the standard cubic Hermite interpolation spline and the quartic Hermite interpolation splines with single parameter by a new algorithm.
\end{abstract}

\section{Introduction}

Spline interpolation is a useful and powerful tool for curves and surfaces modeling. Standard cubic Hermite spline is one of those interpolation functions. But, for the given interpolation condition, the cubic Hermite interpolation spline is fixed; that is to say, the shape of the interpolation curve or surface is fixed for the given interpolation data [1$6]$. Since the interpolation function is unique for the given interpolation data, to modify the shape of the interpolation curve to approximate the given curve seems to be impossible and it is contradictory to the uniqueness of the interpolation function for the given interpolation data. For the given interpolation condition, how to improve the approximation accuracy of the interpolation spline is an important problem in the computer aided geometric design. In recent years, many authors have presented some new method to modify the shape of the interpolation curve to satisfy the industrial product design with several kinds of new interpolation splines with parameters [7-20].

These new splines all have similar properties of the standard cubic Hermite spline. For example, for the given interpolation data, if interpolation interval approaches zero, theoretically speaking, these splines can approximate the given curve and surface well. However, there exists a problem, in the process of the actual calculation; the amount of computation will increase dramatically if the length of the interpolating intervals decreases. On the other hand, the approximation accuracy of these new splines may not be better than the standard cubic Hermite spline.

In [7-17], many rational form interpolation splines with multiple parameters were presented. For the given interpolation data, the change of the parameters causes the change of the interpolation curve. Nevertheless, the computation of the splines with multiple parameters is very complicated. Several kinds of rational splines with a single parameter were presented in the papers $[18,19]$, which is simple to compute, but its approximation accuracy is not good for the given curves and surfaces. In general, polynomial-form splines are suitable to be used to design and compute. In [20], a polynomial-form spline, called quartic Hermite spline with single parameter, is presented as the extension of the standard Hermite spline. The quartic spline has a simple form, and its approximation rate to the given curves and surfaces is not high.

In this paper, a class of new quartic splines with two parameters is developed, which is the extension of the standard cubic Hermite interpolation spline and inherits the same properties of the standard cubic Hermite interpolation spline. For the given interpolation conditions, the shape of the proposed splines can be adjusted by changing the values of the parameters. Furthermore, the introduced splines could 
approximate to the interpolated functions better than the standard cubic Hermite interpolation splines and the quartic Hermite interpolation splines with single parameter.

The remainder of the paper is organized as follows. Section 2 introduces the standard cubic Hermite spline and some of its properties. A kind of interpolation spline with two parameters is presented in Section 3. Section 4 discusses the approximation of the introduced spline curve with numerical examples. In the end, a summary and conclusions are given.

\section{The Standard Cubic Hermite Spline and Its Basis Functions}

Generally, for $t \in[0,1]$, the following four basis functions,

$$
\begin{aligned}
& \alpha_{0}(t)=1-3 t^{2}+2 t^{3}, \\
& \alpha_{1}(t)=3 t^{2}-2 t^{3} \\
& \beta_{0}(t)=t-2 t^{2}+t^{3}, \\
& \beta_{1}(t)=-t^{2}+t^{3}
\end{aligned}
$$

are called the standard cubic Hermite bases.

These bases satisfy

$$
\begin{array}{cc}
\alpha_{0}(0)=\alpha_{1}(1)=1, & \alpha_{0}(1)=\alpha_{1}(0)=0, \\
\alpha_{1}^{\prime}(0)=\alpha_{1}^{\prime}(1)=0, & \alpha_{0}^{\prime}(0)=\alpha_{0}^{\prime}(1)=0, \\
\beta_{0}(0)=\beta_{0}(1)=0, & \beta_{1}(0)=\beta_{1}(1)=0, \\
\beta_{0}^{\prime}(0)=\beta_{1}^{\prime}(1)=1, & \beta_{0}^{\prime}(1)=\beta_{1}^{\prime}(0)=0, \\
\alpha_{0}(t)+\alpha_{1}(t)=1, & \beta_{0}(t)+\beta_{1}(1-t)=0 .
\end{array}
$$

For given knots $a=x_{0}<x_{1}<\cdots<x_{n}=b$ and data $\left\{\left(x_{i}, y_{i}, d_{i}\right), i=0,1, \ldots, n\right\}$, where $y_{i}$ and $d_{i}$ are the values of the function value and the first-order derivative value of the function being interpolated, let $h_{i}=x_{i+1}-x_{i}, t=\left(x-x_{i}\right) / h_{i}$ and then the standard cubic Hermite spline in the interval $\left[x_{i}, x_{i+1}\right]$ can be defined as follows:

$$
\begin{array}{r}
H_{i}(x)=\alpha_{0}(t) y_{i}+\alpha_{1}(t) y_{i+1}+\beta_{0}(t) h_{i} d_{i}+\beta_{1}(t) h_{i} d_{i+1}, \\
i=0,1, \ldots, n-1 .
\end{array}
$$

Obviously, we have $H_{i}\left(x_{i}\right)=y_{i}, H_{i}\left(x_{i+1}\right)=y_{i+1}, H_{i}^{\prime}\left(x_{i}\right)=$ $d_{i}, H_{i}^{\prime}\left(x_{i+1}\right)=d_{i+1}$.

The standard cubic Hermite spline is $C^{1}$ continuous. However, if interpolation data is given, the shape and approximation of the spline cannot be changed.

\section{The EH Interpolation Spline}

In order to overcome the disadvantage of the standard cubic Hermite spline, we extend its basis functions firstly.

\subsection{The Basis Functions of the EH Interpolation Spline}

Definition 1. For arbitrary real number $\lambda_{i}, \mu_{i}$ and $0 \leq t \leq 1$, the following four functions with parameters $\lambda_{i}, \mu_{i}$,

$$
\begin{aligned}
& e \alpha_{0}(t)=1+\left(\lambda_{i}-3\right) t^{2}+2\left(1-\lambda_{i}\right) t^{3}+\lambda_{i} t^{4}, \\
& e \alpha_{1}(t)=\left(3-\lambda_{i}\right) t^{2}+2\left(\lambda_{i}-1\right) t^{3}-\lambda_{i} t^{4}, \\
& e \beta_{0}(t)=t+\left(\mu_{i}-2\right) t^{2}+\left(1-2 \mu_{i}\right) t^{3}+\mu_{i} t^{4}, \\
& e \beta_{1}(t)=-\left(\mu_{i}+1\right) t^{2}+\left(1+2 \mu_{i}\right) t^{3}-\mu_{i} t^{4},
\end{aligned}
$$

are calledbasis functions of the $\mathrm{EH}$ interpolation spline, briefly EH bases.

The EH bases are the extension of the standard cubic Hermite bases. When $\lambda_{i}=\mu_{i}=0$, the bases are the standard cubic Hermite bases. The bases have the similar properties of the standard cubic Hermite bases.

By computing directly, we have $e \alpha_{0}(0)=e \alpha_{1}(1)=$ $1, e \alpha_{0}(1)=e \alpha_{1}(0)=0, e \alpha_{1}^{\prime}(0)=e \alpha_{1}^{\prime}(1)=0, e \alpha_{0}^{\prime}(0)=$ $e \alpha_{0}^{\prime}(1)=0, e \beta_{0}(0)=e \beta_{0}(1)=0, e \beta_{1}(0)=e \beta_{1}(1)=$ $0, e \beta_{0}^{\prime}(0)=e \beta_{1}^{\prime}(1)=1, e \beta_{1}^{\prime}(1)=e \beta_{1}^{\prime}(0)=0$, and $e \alpha_{0}(t)+$ $e \alpha_{1}(t)=1, e \beta_{0}(t)+e \beta_{1}(1-t)=0$.

When $\lambda_{i}=\mu_{i}$, the EH bases (4) are basis functions with single parameter in [20].

Figure 1 shows the four EH bases, where the solid lines are the standard Hermite bases, the parameters $\lambda_{i}=2, \mu_{i}=-2$ are for the dot-dash lines, and $\lambda_{i}=-2, \mu_{i}=2$ are for dashed line.

So, we may construct the Ferguson curve with two parameters based on the $\mathrm{EH}$ bases as follows:

$$
\mathrm{EH}_{i}(t)=e \alpha_{0}(t) p_{i}+e \alpha_{1}(t) p_{i+1}+e \beta_{0} p_{i}^{\prime}+e \beta_{1}(t) p_{i+1}^{\prime},
$$

where $p_{i}, p_{i+1}$ and $p_{i}^{\prime}, p_{i+1}^{\prime}$ are two interpolation points and their tangent vectors, respectively.

For the given two interpolation points and tangent vectors, with the different parameters $\lambda_{i}, \mu_{i}$, we may obtain different shape of the Ferguson curve with two parameters accordingly.

Figure 2 shows the Ferguson curves with different parameters, where the solid line is the standard Ferguson spline curve with $\lambda_{i}=\mu_{i}=0$, the parameters $\lambda_{i}=2, \mu_{i}=-2$ are for the dot-dash line, and $\lambda_{i}=-2, \mu_{i}=2$ are for dashed line.

\subsection{The EH Interpolation Spline}

Definition 2. Given a data set $\left\{\left(x_{i}, y_{i}, d_{i}\right), i=0,1, \ldots, n\right\}$, where $y_{i}$ and $d_{i}$ are the values of the function value and the first-order derivative value of the function being interpolated and $a=x_{0}<x_{1}<\cdots<x_{n}=b$ is the knot spacing, let 


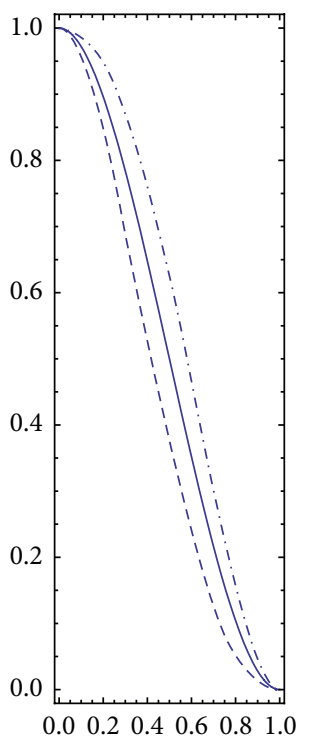

(a) $e \alpha_{0}(t)$

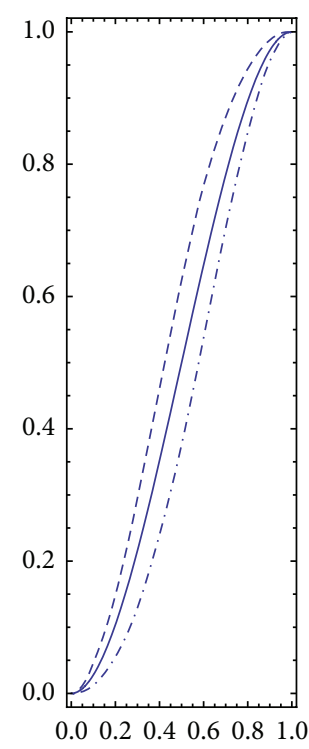

(b) $e \alpha_{1}(t)$

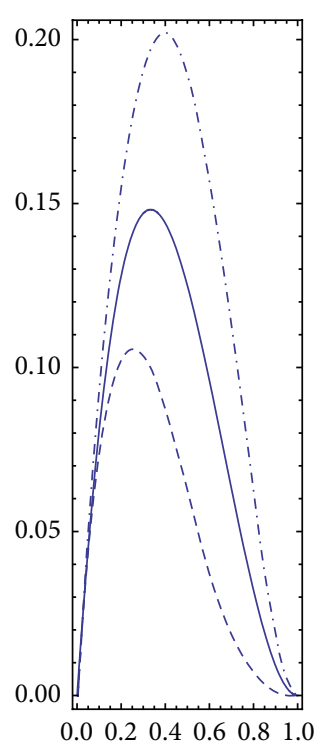

(c) $e \beta_{0}(t)$

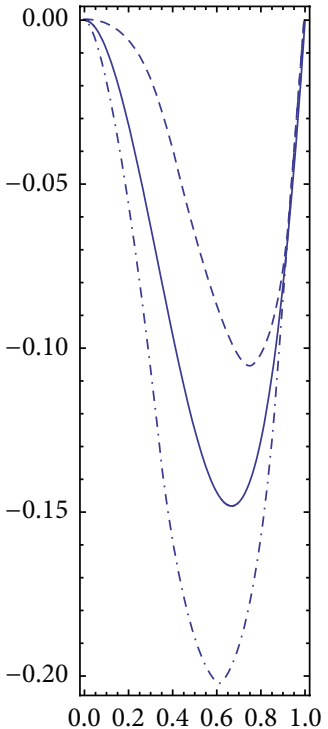

(d) $e \beta_{1}(t)$

FIGURE 1: The graph of the four EH bases.

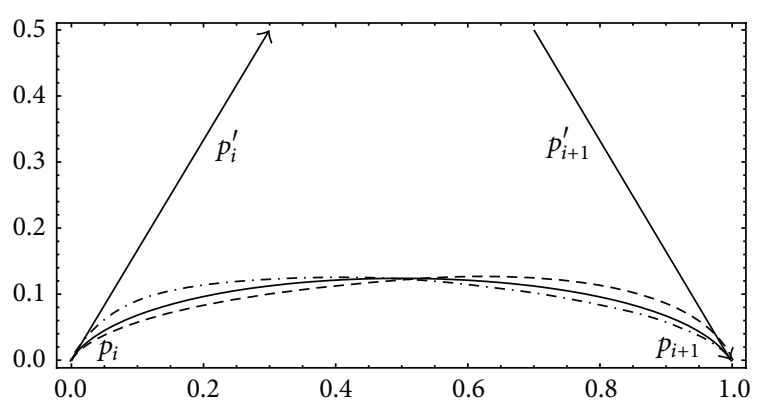

FIgURE 2: The Ferguson curve with different parameters.

$h_{i}=x_{i+1}-x_{i}, t=\left(x-x_{i}\right) / h_{i}$; then the EH interpolation spline in the interval $\left[x_{i}, x_{i+1}\right]$ can be defined as follows:

$$
\begin{array}{r}
\left.\mathrm{EH}(x)\right|_{\left[x_{i}, x_{i+1}\right]} \\
=y_{i} e \alpha_{0}(t)+y_{i+1} e \alpha_{1}(t)+d_{i} h_{i} e \beta_{0}(t)+d_{i+1} h_{i} e \beta_{1}(t), \\
i=0,1, \ldots, n-1,
\end{array}
$$

where $e \alpha_{0}(t), e \alpha_{1}(t), e \beta_{0}(t), e \beta_{1}(t)$ are the $\mathrm{EH}$ bases.

Obviously, for the data set $\left\{\left(x_{i}, y_{i}, d_{i}\right), i=0,1, \ldots, n\right\}$, $\mathrm{EH}(x)$ satisfies

$$
\begin{aligned}
& \mathrm{RH}\left(x_{i}\right)=y_{i}, \quad \operatorname{RH}^{\prime}\left(x_{i}\right)=d_{i} \text {, } \\
& i=0,1, \ldots, n \text {. }
\end{aligned}
$$

If $\lambda_{i}=\mu_{i}=0$, it is just the standard cubic Hermite spline. It is of interest that, for suitable selected parameters $\lambda_{i}, \mu_{i}$, the piecewise interpolation function $\mathrm{EH}(x)$ can be
$C^{2}$-continuous in the interval $\left[x_{0}, x_{n}\right]$. In fact, denote $\Delta_{i}=$ $\left(y_{i+1}-y_{i}\right) / h_{i}$, and let

$$
\mathrm{EH}^{\prime \prime}\left(x_{i}+\right)=\mathrm{EH}^{\prime \prime}\left(x_{i}-\right), \quad i=1,2, \ldots, n-1 ;
$$

then the equations connecting the parameters $\lambda_{i}$ and $\mu_{i}$,

$$
\begin{aligned}
h_{i} & {\left[\Delta_{i-1}\left(\lambda_{i-1}+3\right)-\left(d_{i}\left(2-\mu_{i-1}\right)+d_{i-1}\left(\mu_{i-1}+1\right)\right)\right] } \\
& =h_{i-1}\left[\Delta_{i}\left(\lambda_{i}-3\right)+\left(d_{i+1}\left(1+\mu_{i}\right)+d_{i}\left(2-\mu_{i}\right)\right)\right],
\end{aligned}
$$

$$
i=1,2, \ldots, n-1 \text {, }
$$

may be obtained. If the successive parameters $\left(\lambda_{i-1}, \mu_{i-1}\right)$ and $\left(\lambda_{i}, \mu_{i}\right)$ satisfy $(9)$ at $i=1,2, \ldots, n-1$, then $\operatorname{EH}(x) \in$ $C^{2}\left(x_{0}, x_{n}\right)$. Furthermore, if the knots are equally spaced and $\lambda_{i}=\mu_{i}=0$, then (9) becomes the well-known tridiagonal system for a cubic spline

$$
d_{i-1}+4 d_{i}+d_{i+1}=3\left(\Delta_{i-1}+\Delta_{i}\right), \quad i=1,2, \ldots, n-1 .
$$

Hence, if given the parameter values $\lambda_{0}, \mu_{0}$ in the interval $\left[x_{0}, x_{1}\right]$, by (9), we may obtain the $\lambda_{1}$ and $\mu_{1}$ and so on. Thus, we can construct a $C^{2}$-continuous interpolation curve.

\section{The Approximation of the EH Interpolation Spline}

According to the interpolation reminder of cubic Hermite spline, when interpolation interval approaches zero, the cubic Hermite spline curve can approximate well to the function being interpolated. However, for the $\mathrm{EH}$ interpolation spline we constructed, it can approximate well to the function being 
TABLE 1: The parameters $\lambda_{i}$ and $\mu_{i}$ for EH interpolation spline and the max error.

\begin{tabular}{lcccccc}
\hline$x_{i}$ & $y_{i}$ & $d_{i}$ & $\lambda_{i}$ & $\mu_{i}$ & $R_{H}$ & $H \varepsilon_{i}$ \\
\hline 0.0000 & 1.0000 & 1.0000 & 0.0421 & 0.0412 & $0.2569 \times 10^{-4}$ & $0.9062 \times 10^{-3}$ \\
0.5000 & 1.2071 & -0.1107 & 0.0146 & 0.0129 & $0.2111 \times 10^{-4}$ & $0.2569 \times 10^{-3}$ \\
1.0000 & 1.0000 & -0.5708 & 0.2451 & 0.2783 & $0.3769 \times 10^{-4}$ & $0.2569 \times 10^{-3}$ \\
1.5000 & 0.7955 & -0.1451 & 0.0188 & 0.0192 & $0.2974 \times 10^{-4}$ & $0.1069 \times 10^{-2}$ \\
2.0000 & 1.0000 & 1.0000 & 0.0108 & 0.0108 & $0.1735 \times 10^{-4}$ & $0.8647 \times 10^{-3}$ \\
2.5000 & 1.7929 & 2.1107 & & & \\
\hline
\end{tabular}

interpolated without interpolation interval approaching zero, and it can approximate to the interpolated functions better than the standard cubic Hermite interpolation spline.

Firstly, we give the definition of the "good approximation."

Definition 3. Let $H_{i}(x)$ be the standard cubic Hermite spline, $\mathrm{EH}_{i}(x)$ be the $\mathrm{EH}$ interpolation spline, and $y(x)$ be the function being interpolated. Denoting $\mathrm{EH}_{i}=$ $\max _{x_{i}<x<x_{i+1}}\left|\mathrm{EH}_{i}(x)-y(x)\right|, H \varepsilon_{i}=\max _{x_{i}<x<x_{i+1}} \mid H_{i}(x)-$ $y(x) \mid$, then if $\mathrm{EH}_{i}<H \varepsilon_{i}$, we can call $\mathrm{RH}_{i}(x)$ has "good approximation" to the interpolated function $y(x)$ better than $H_{i}(x)$.

According to the Definition 3, if $\mathrm{EH}_{i}<H \varepsilon_{i}$, we can get the range of the parameters value, $\lambda_{i}$ and $\mu_{i}$. In the range of the parameters value, selecting the arbitrary values of the parameters $\lambda_{i}$ and $\mu_{i}$, we have a "good approximation" curve.

Example 4. Given the function $y(x)=x+\cos ((\pi / 2) x)$ and knots $x_{i}=(i / 2)(i=0,1, \ldots, 5)$, namely, $h_{i}=(i / 2)(i=$ $0, \ldots, 4)$. According to the inequality $\mathrm{EH} \varepsilon_{i}<H \varepsilon_{i}$, we may get the range of the parameters $\lambda_{i}$ and $\mu_{i}$. For the fixed interpolation condition, the max error and the parameters $\lambda_{i}$

and $\mu_{i}$ are given for every interval $\left[x_{i}, x_{i+1}\right]$ in Table 1 . The error curves of the $\operatorname{EH}(x)$ and $H(x)$ to $y(x)$ are shown in Figure 3.

By using the tensor product method, we can construct the $\mathrm{EH}$ interpolation spline surfaces, which has the similar $\mathrm{EH}$ interpolation spline curve.

Definition 5. Let $\Omega:[a, b] \times[c, d]$ be the plane region and $f(x, y)$ a bivariate function defined in the region $\Omega$ and let $a=x_{0}<x_{1}<\cdots<x_{m}=b$ and $c=y_{0}<y_{1}<\cdots<y_{n}=$ $d$ be the knot sequences. Denote $h_{i}=x_{i+1}-x_{i}, h_{j}=y_{j+1}-y_{j}$, $u=\left(x-x_{i}\right) / h_{i}, v=\left(y-y_{j}\right) / h_{j}$; then the EH interpolation spline surface on the region $\left[x_{i}, x_{i+1}\right] \times\left[y_{i}, y_{i+1}\right]$ can be defined as follows:

$$
\begin{aligned}
\mathrm{EH} & \left.(x, y)\right|_{\left.x_{i}, x_{i+1}\right]} ^{\left[y_{i}, y_{i+1}\right]} \\
& =\left(e \alpha_{0}(u), e \alpha_{1}(u), e \beta_{0}(u), e \beta_{1}(u)\right) M\left(\begin{array}{l}
e \alpha_{0}(v) \\
e \alpha_{1}(v) \\
e \beta_{0}(v) \\
e \beta_{1}(v)
\end{array}\right),
\end{aligned}
$$

where

$$
M=\left(\begin{array}{cccc}
f\left(x_{i}, y_{i}\right) & f\left(x_{i}, y_{i+1}\right) & h_{j} f_{v}^{\prime}\left(x_{i}, y_{i}\right) & h_{j} f_{v}^{\prime}\left(x_{i}, y_{i+1}\right) \\
f\left(x_{i+1}, y_{i}\right) & f\left(x_{i+1}, y_{i+1}\right) & h_{j} f_{v}^{\prime}\left(x_{i+1}, y_{i}\right) & h_{j} f_{v}^{\prime}\left(x_{i+1}, y_{i+1}\right) \\
h_{i} f_{u}^{\prime}\left(x_{i}, y_{i}\right) & h_{i} f_{u}^{\prime}\left(x_{i}, y_{i+1}\right) & h_{i} h_{j} f_{u v}^{\prime \prime}\left(x_{i}, y_{i}\right) & h_{i} h_{j} f_{u v}^{\prime \prime}\left(x_{i}, y_{i+1}\right) \\
h_{i} f_{u}^{\prime}\left(x_{i+1}, y_{i}\right) & h_{i} f_{u}^{\prime}\left(x_{i+1}, y_{i+1}\right) & h_{i} h_{j} f_{u v}^{\prime \prime}\left(x_{i+1}, y_{i}\right) & h_{i} h_{j} f_{u v}^{\prime \prime}\left(x_{i+1}, y_{i+1}\right)
\end{array}\right) .
$$

Given the end-points, the first order partial derivative and the second-order blending partial derivative of the function interpolated, with proper parameters, the $\mathrm{EH}$ interpolation spline surfaces could approximate to the bivariate functions being interpolated better than the standard cubic Hermite spline surfaces.

Example 6. Given the bivariate function being interpolated $f(x, y)=\sin (\pi / 2) x \cos (\pi / 2) y$, let $a=0<1<2=b$ and $c=$ $-1<0<1=d$ be the knot sequences. Denote $h_{i}=x_{i+1}-x_{i}$, $h_{j}=y_{j+1}-y_{j}, u=\left(x-x_{i}\right) / h_{i}$, and $v=\left(y-y_{j}\right) / h_{j}$. By selecting $\lambda_{0}=\lambda_{1}=0.3208, \mu_{0}=\mu_{1}=0.6995$, we can work out that the max error of the $\mathrm{EH}(x, y)-f(x, y)$ equals $0.5069 \times 10^{-3}$, but the max error of the $H(x, y)-f(x, y)$ equals $0.1061 \times 10^{-1}$.

Figure 4 shows the error surface of the $\mathrm{EH}(x, y)-f(x, y)$. Figure 5 shows the error surface of the $H(x, y)-f(x, y)$.

\section{Conclusion}

This paper introduced a kind of $\mathrm{EH}$ interpolation spline, which is the extension of the standard cubic Hermite interpolation spline. The shape of the proposed splines can be adjusted by changing the values of the parameters for the 


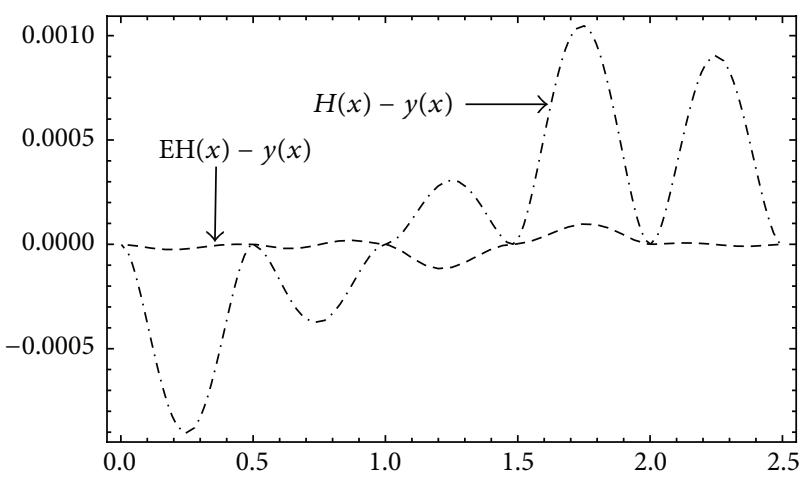

FIgURE 3: The error curves of the $\mathrm{EH}(x)-y(x)$ and $H(x)-y(x)$.

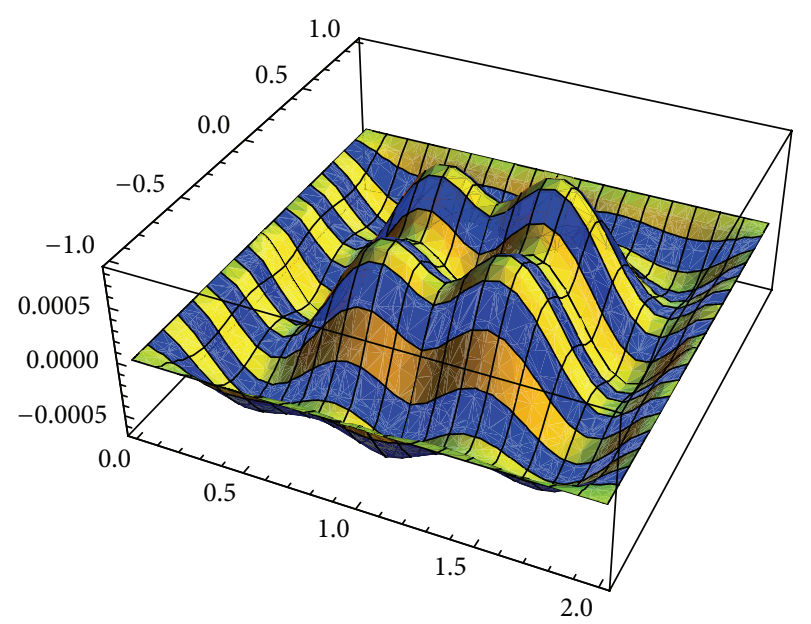

FIgURE 4: The error surface of the $\operatorname{EH}(x, y)-f(x, y)$.

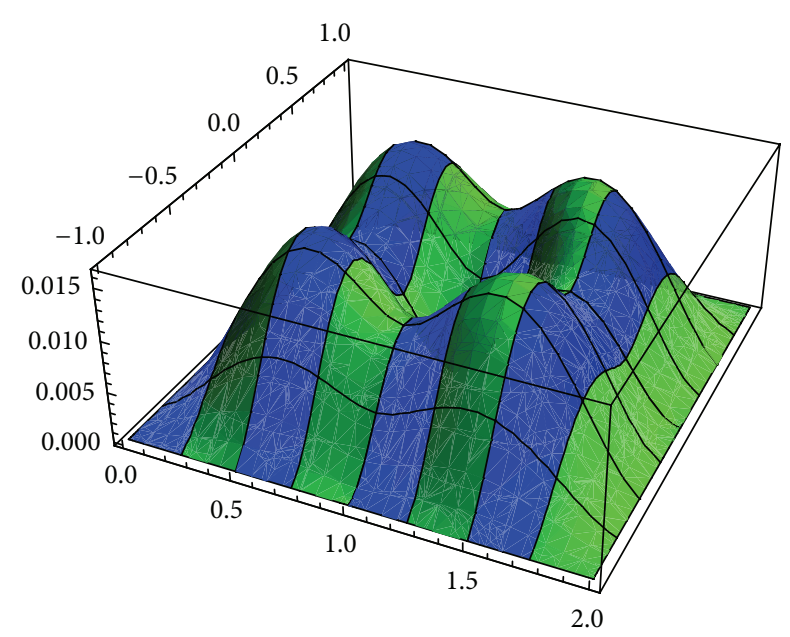

Figure 5: The error surface of the $H(x, y)-f(x, y)$.

fixed interpolation conditions. Also, the introduced spline could approximate to the interpolated function better than the standard cubic Hermite interpolation spline.

\section{Conflict of Interests}

The authors declare that there is no conflict of interests regarding the publication of this paper.

\section{Acknowledgments}

The work was funded by the Natural Science Foundation of Anhui Province of China under Grant no. 1208085MA15, the Key Project Foundation of Scientific Research, Education Department of Anhui Province under Grant no. KJ2014ZD30, and the Key Construction Disciplines Foundation of Hefei University under Grant no. 2014XK08.

\section{References}

[1] S. Butt and K. W. Brodlie, "Preserving positivity using piecewise cubic interpolation," Computers \& Graphics, vol. 17, no. 1, pp. 5564, 1993.

[2] K. W. Brodlie and S. Butt, "Preserving convexity using piecewise cubic interpolation," Computers and Graphics, vol. 15, no. 1, pp. $15-23,1991$.

[3] R. L. Dougherty, A. S. Edelman, and J. M. Hyman, "Nonnegativity, monotonicity, or convexity-preserving cubic and quintic Hermite interpolation," Mathematics of Computation, vol. 52, no. 186, pp. 471-494, 1989.

[4] R. E. Carlson and F. N. Fritsch, "Monotone piecewise cubic interpolation," SIAM Journal on Numerical Analysis, vol. 17, no. 2, pp. 238-246, 1980.

[5] F. N. Fritsch and J. Butland, "A method for constructing local monotone piecewise cubic interpolants," SIAM Journal on Scientific and Statistical Computation, vol. 5, no. 2, pp. 300-304, 1984.

[6] L. L. Schumaker, "On shape preserving quadratic spline interpolation," SIAM Journal on Numerical Analysis, vol. 20, no. 4, pp. 854-864, 1983.

[7] Q. Duan, Y. Zhang, L. Wang, and E. H. Twizell, "Region control and approximation of a weighted rational interpolating curves," Communications in Numerical Methods in Engineering, vol. 22, no. 1, pp. 41-53, 2006.

[8] Q. I. Duan, K. Djidjeli, W. G. Price, and E. H. Twizell, "A rational cubic spline based on function values," Computers and Graphics, vol. 22, no. 4, pp. 479-486, 1998.

[9] Q. Duan, K. Djidjeli, W. G. Price, and E. H. Twizell, "The approximation properties of some rational cubic splines," International Journal of Computer Mathematics, vol. 72, no. 2, pp. 155-166, 1999.

[10] M. Sarfraz, "Cubic spline curves with shape control," Computers and Graphics, vol. 18, no. 5, pp. 707-713, 1994.

[11] Q. Duan, A. K. Liu, and F. H. Cheng, "Constrained interpolation using rational cubic spline with linear denominators," The Korean Journal of Computational \& Applied Mathematics. An International Journal, vol. 6, no. 1, pp. 203-215, 1999.

[12] M. Z. Hussain and M. Sarfraz, "Positivity-preserving interpolation of positive data by rational cubics," Journal of Computational and Applied Mathematics, vol. 218, no. 2, pp. 446-458, 2008.

[13] M. Sarfraz, M. Z. Hussain, and M. Hussain, "Shape-preserving curve interpolation," International Journal of Computer Mathematics, vol. 89, no. 1, pp. 35-53, 2012. 
[14] F. Ibraheem, M. Hussain, M. Z. Hussain, and A. A. Bhatti, "Positive data visualization using trigonometric function," Journal of Applied Mathematics, vol. 2012, Article ID 247120, 19 pages, 2012.

[15] Q. Duan, H. Zhang, Y. Zhang, and E. H. Twizell, "Error estimation of a kind of rational spline," Journal of Computational and Applied Mathematics, vol. 200, no. 1, pp. 1-11, 2007.

[16] M. Tian and H. L. Geng, "Error analysis of a rational interpolation spline," International Journal of Mathematical Analysis, vol. 5, no. 25-28, pp. 1287-1294, 2011.

[17] F. Bao, Q. Sun, J. Pan, and Q. Duan, "Point control of rational interpolating curves using parameters," Mathematical and Computer Modelling, vol. 52, no. 1-2, pp. 143-151, 2010.

[18] J. Xie, J. Q. Tan, and S. F. Li, "Rational cubic Hermite interpolating spline and its approximation properties," Chinese Journal of Engineering Mathematics, vol. 28, no. 3, pp. 385-392, 2010.

[19] J. Xie, J. Q. Tan, and S. F. Li, "A kind of rational cubic spline and its applications," Acta Mathematicae Applicatae Sinica, vol. 23, no. 35, pp. 847-855, 2010.

[20] C.-Y. Liu, L. Yang, and J.-C. Li, "Quartic Hermite interpolating splines with parameters," Journal of Computer Applications, vol. 32, no. 7, pp. 1868-1870, 2012. 


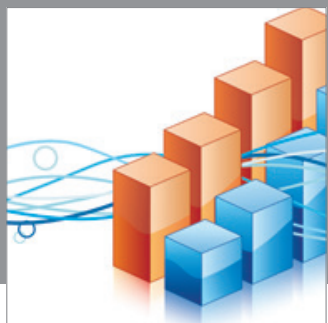

Advances in

Operations Research

mansans

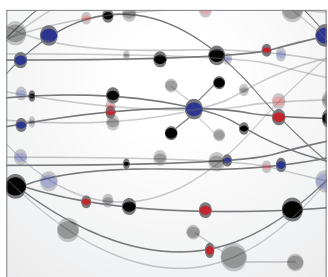

The Scientific World Journal
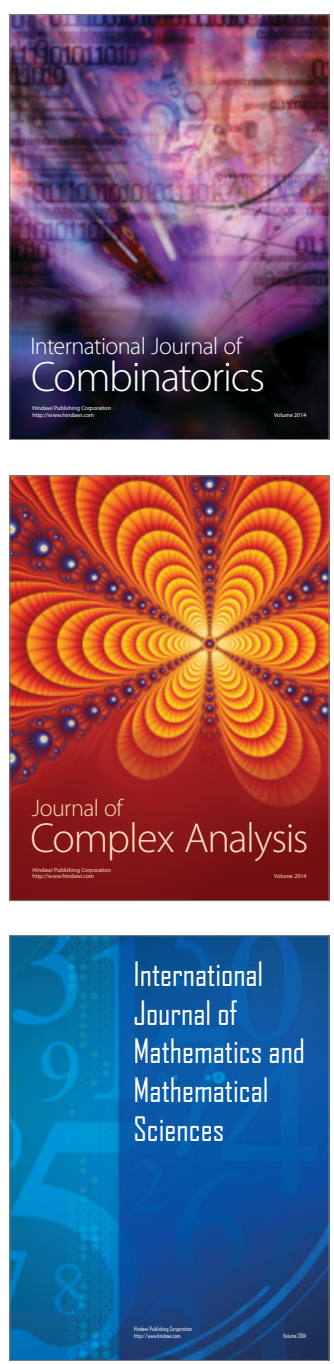
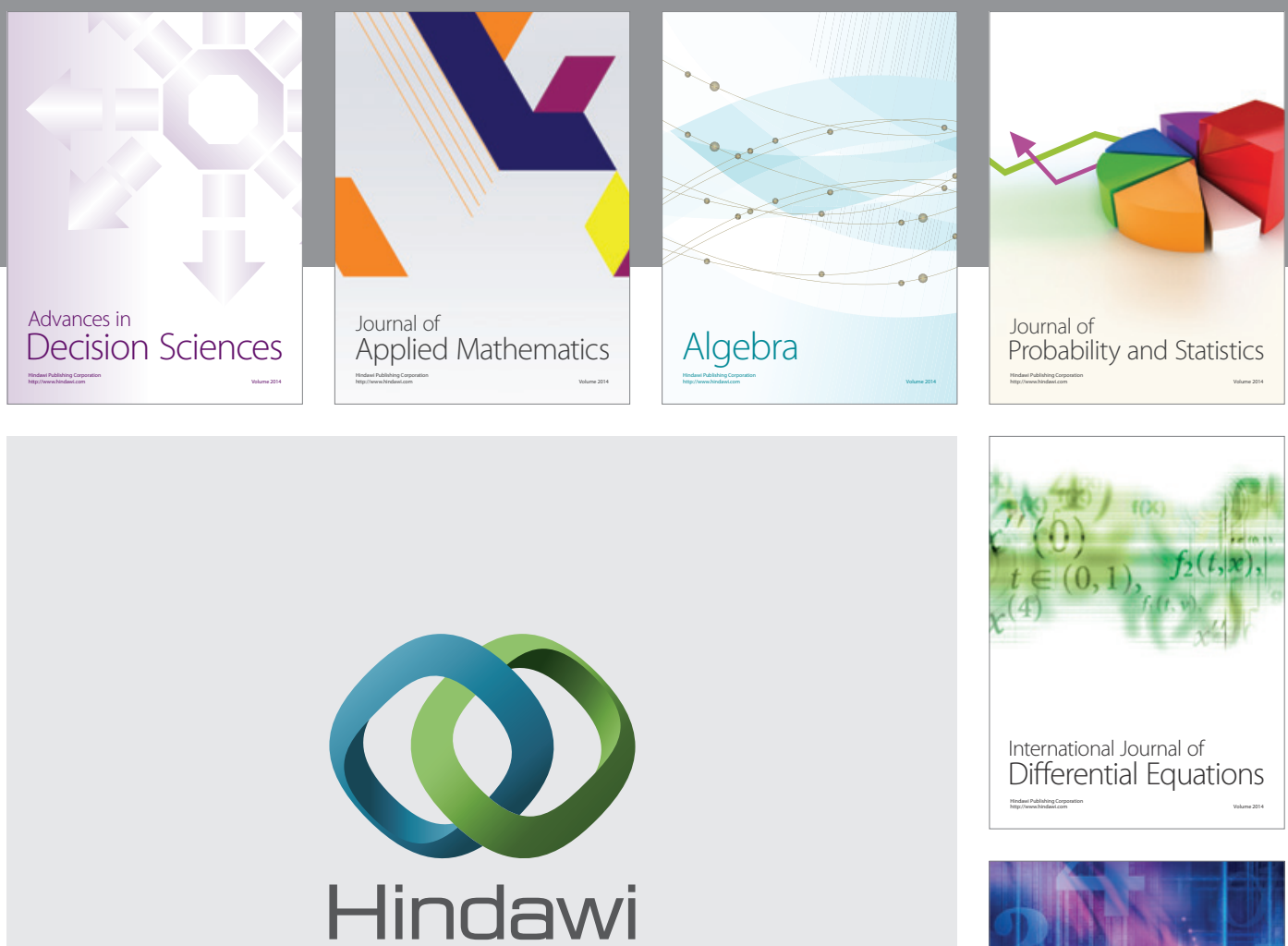

Submit your manuscripts at http://www.hindawi.com
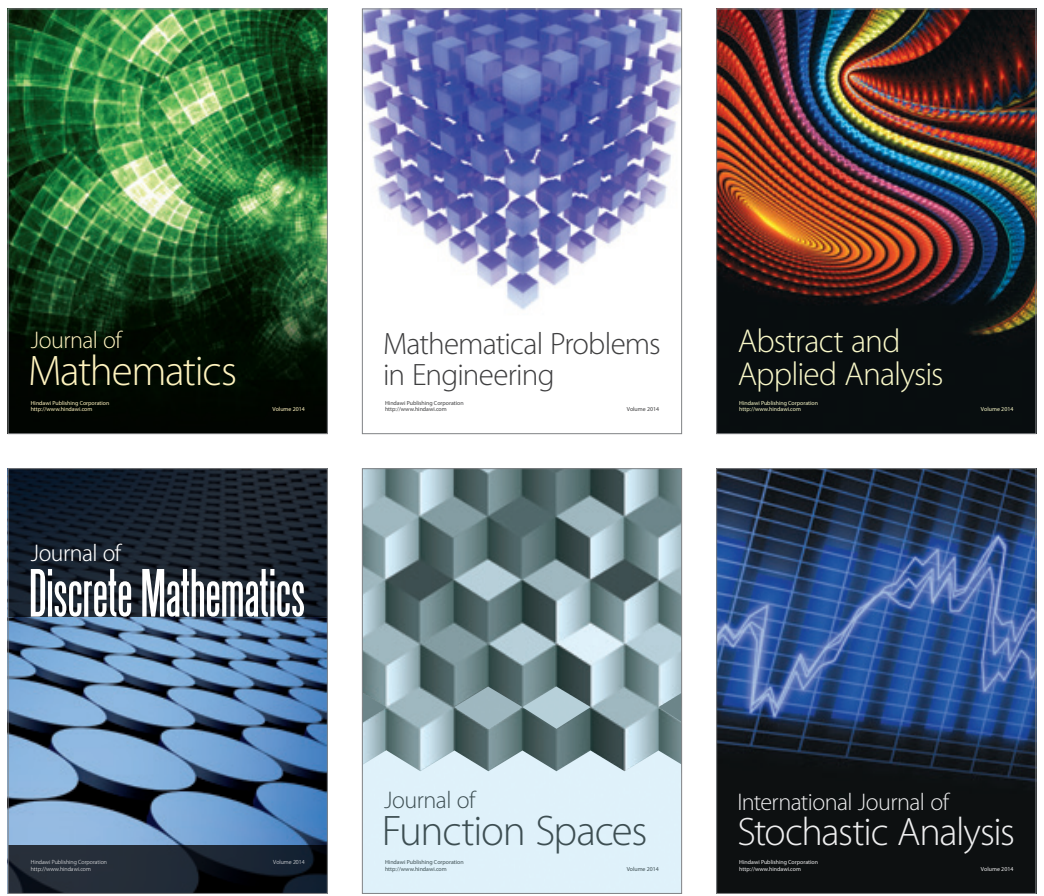

Journal of

Function Spaces

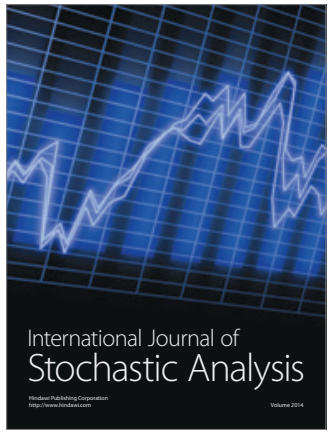

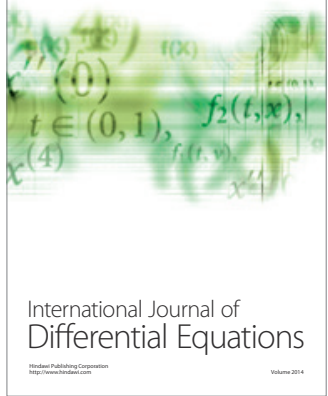
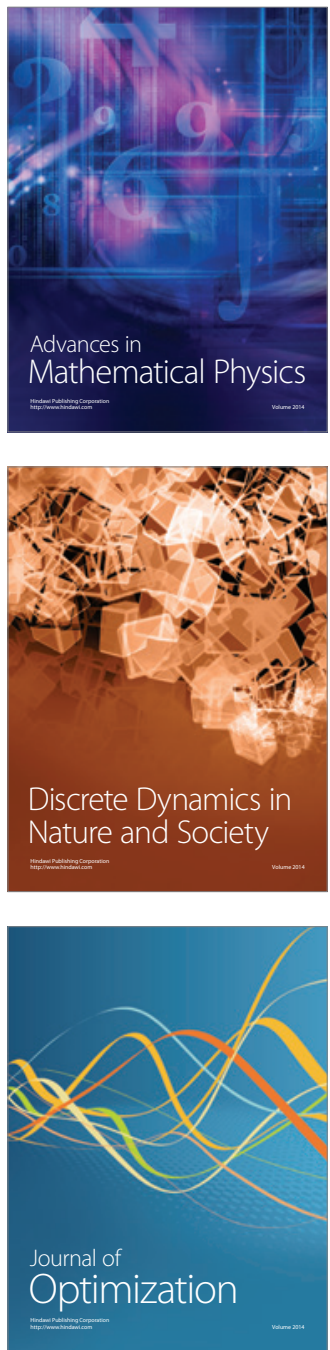